\title{
An unidentified TeV source in the vicinity of Cygnus OB2
}

\author{
F. Aharonian ${ }^{1}$, A. Akhperjanian ${ }^{7}$, M. Beilicke ${ }^{4}$, K. Bernlöhr ${ }^{1}$, H. Börst ${ }^{5}$, H. Bojahr ${ }^{6}$, O. Bolz ${ }^{1}$, T. Coarasa ${ }^{2}$,
} J. Contreras ${ }^{3}$, J. Cortina ${ }^{2}$, S. Denninghoff ${ }^{2}$, V. Fonseca ${ }^{3}$, M. Girma ${ }^{1}$, N. Götting ${ }^{4}$, G. Heinzelmann ${ }^{4}$, G. Hermann ${ }^{1}$, A. Heusler ${ }^{1}$, W. Hofmann ${ }^{1}$, D. Horns ${ }^{1}$, I. Jung ${ }^{1}$, R. Kankanyan ${ }^{1}$, M. Kestel ${ }^{2}$, J. Kettler ${ }^{1}$, A. Kohnle ${ }^{1}$, A. Konopelko ${ }^{1}$, H. Kornmeyer ${ }^{2}$, D. Kranich ${ }^{2}$, H. Krawczynski ${ }^{9}$, H. Lampeitl ${ }^{4}$, M. Lopez ${ }^{3}$, E. Lorenz ${ }^{2}$, F. Lucarelli ${ }^{3}$, N. Magnussen ${ }^{10}$, O. Mang ${ }^{5}$, H. Meyer ${ }^{6}$, M. Milite ${ }^{4}$, R. Mirzoyan ${ }^{2}$, A. Moralejo ${ }^{3}$, E. Ona ${ }^{3}$, M. Panter ${ }^{1}$, A. Plyasheshnikov ${ }^{1,8}$, J. Prahl $^{4}$, G. Pühlhofer ${ }^{1}$, G. Rauterberg ${ }^{5}$, R. Reyes ${ }^{2}$, W. Rhode ${ }^{6}$, J. Ripken ${ }^{4}$, A. Röhring ${ }^{4}$, G. P. Rowell ${ }^{1}$, V. Sahakian ${ }^{7}$, M. Samorski ${ }^{5}$, M. Schilling ${ }^{5}$, F. Schröder ${ }^{6}$, M. Siems ${ }^{5}$, D. Sobzynska ${ }^{2}$, W. Stamm ${ }^{5}$, M. Tluczykont ${ }^{4}$, H. J. Völk ${ }^{1}$, C. A. Wiedner ${ }^{1}$, W. Wittek ${ }^{2}$ (HEGRA Collaboration), Y. Uchiyama ${ }^{11}$, and T. Takahashi ${ }^{11}$

1 Max-Planck-Institut für Kernphysik, Postfach 103980, 69029 Heidelberg, Germany

2 Max-Planck-Institut für Physik, Föhringer Ring 6, 80805 München, Germany

3 Universidad Complutense, Facultad de Ciencias Físicas, Ciudad Universitaria, 28040 Madrid, Spain

${ }^{4}$ Universität Hamburg, Institut für Experimentalphysik, Luruper Chaussee 149, 22761 Hamburg, Germany

5 Universität Kiel, Institut für Experimentelle und Angewandte Physik, Leibnizstraße 15-19, 24118 Kiel, Germany

${ }^{6}$ Universität Wuppertal, Fachbereich Physik, Gaußstr. 20, 42097 Wuppertal, Germany

7 Yerevan Physics Institute, Alikhanian Br. 2, 375036 Yerevan, Armenia

8 On leave from Altai State University, Dimitrov Street 66, 656099 Barnaul, Russia

9 Now at Washington University, St. Louis, MO 63130, USA

10 Now at IFAE, Unversitat Autònoma de Barcelona, Spain

11 Institute of Space and Astronautical Science, 3-1-1 Yoshinodai, Sagamihara, Kanagawa 229-8510, Japan

Received 24 July 2002 / Accepted 9 August 2002

\begin{abstract}
Deep observation ( $113 \mathrm{hrs})$ of the Cygnus region at TeV energies using the HEGRA stereoscopic system of air Čerenkov telescopes has serendipitously revealed a signal positionally inside the core of the OB association Cygnus OB2, at the edge of the $95 \%$ error circle of the EGRET source 3EG J2033+4118, and $\sim 0.5^{\circ}$ north of Cyg X-3. The source centre of gravity is RA $\alpha_{\mathrm{J} 2000}: 20^{\mathrm{hr}} 32^{\mathrm{m}} 07^{\mathrm{s}} \pm 9.2_{\text {stat }}^{\mathrm{s}} \pm 2.2_{\text {sys }}^{\mathrm{s}}$, Dec $\delta_{\mathrm{J} 2000}:+41^{\circ} 30^{\prime} 30^{\prime \prime} \pm 2.0_{\text {stat }}^{\prime} \pm 0.4_{\text {sys }}^{\prime}$. The source is steady, has a post-trial significance of $+4.6 \sigma$, indication for extension with radius $5.6^{\prime}$ at the $\sim 3 \sigma$ level, and has a differential power-law flux with hard photon index of $-1.9 \pm 0.3_{\text {stat }} \pm 0.3_{\text {sys }}$. The integral flux above $1 \mathrm{TeV}$ amounts $\sim 3 \%$ that of the Crab. No counterpart for the TeV source at other wavelengths is presently identified, and its extension would disfavour an exclusive pulsar or AGN origin. If associated with Cygnus OB2, this dense concentration of young, massive stars provides an environment conducive to multi-TeV particle acceleration and likely subsequent interaction with a nearby gas cloud. Alternatively, one could envisage $\gamma$-ray production via a jet-driven termination shock.
\end{abstract}

Key words. gamma rays: observations - stars: early-type - Galaxy: open clusters and associations: individual: Cygnus OB2

\section{Introduction}

The current generation of ground-based imaging atmospheric Čerenkov telescopes offer coverage of the multi $\mathrm{GeV}$ to $\mathrm{TeV}$ $\gamma$-ray sky at centi-Crab sensitivity and arc-minute resolution. Stereoscopy employed by the HEGRA CT-System at La Palma (Daum et al. 1997) offers highly accurate reconstruction of event directions at angles up to $\sim 3^{\circ}$ off-axis. Results here are taken from data originally devoted to Cyg X-3, and the EGRET source GeV J2035+4214 (Lamb \& Macomb 1997). The separation between these objects $\left(\sim 1.5^{\circ}\right)$ permits a

Send offprint requests to: G. P. Rowell, D. Horns e-mail: Gavin. Rowell@mpi-hd.mpg. de, Dieter.Horns@mpi-hd.mpg.de combined analysis given the overlap in their CT-System fields of view (FOV). This letter presents analysis details and observational properties of a serendipitously discovered $\mathrm{TeV}$ source in these data. A brief discussion concerning astrophysical origin and location of this new source is also presented.

\section{Data analysis and results}

The HEGRA system of imaging atmospheric Čerenkov telescopes (IACT-System), consists of 5 identical telescopes operating in coincidence for the stereoscopic detection of air showers induced by primary $\gamma$-rays in the atmosphere. In data dedicated to Cyg X-3, alternate $\sim 20$ min runs targeting the Cyg X-3 position $\pm 0.5^{\circ}$ in declination were taken during 
moonless nights of Aug.-Sept. 1999, Sept.-Oct. 2000 and Jun.Oct. 2001. Likewise in data dedicated to $\mathrm{GeV} \mathrm{J2035+4124,}$ $\sim 20$ min runs were obtained tracking directly the GeV source during Jul.-Aug. 2001. In total, three tracking positions are present in combined data. After quality checks, a total of 112.9 hours data are available for analysis. Preferential selection of $\gamma$-ray-like events (against the cosmic-ray background) is achieved by using the difference between the reconstructed and assumed event direction, $\theta$, and the mean-scaled-width parameter, $\bar{w}$ (Konopelko 1995). In searching for weak point-like and marginally extended sources, so-called tight cuts are considered optimal given the angular resolution of the CT-System $\left(<0.1^{\circ}\right): \theta<0.12^{\circ}$ and $\bar{w}<1.1$, where we use algorithm " 3 " as described by Hofmann et al. (1999) for the event direction reconstruction. The number of images per event, $n_{\text {tel }}$, used for calculating $\theta$ and $\bar{w}$ was also a priori chosen at $n_{\text {tel }} \geq 3$. Monte Carlo simulations (Konopelko et al. 1999) and tests on real sources have shown that $n_{\text {tel }}=2$ events contribute little to the overall sensitivity.

\subsection{Source search and background estimates}

In searching for new TeV sources, skymaps of event direction excesses over the RA and Dec plane are generated after having estimated the background over the FOV. A new empirically-based template background model has been developed with the goal of simple generation of skymaps. The template background comprises events normally rejected according to the $\bar{w}$ criterion. We define the number of events in the $\gamma$-ray regime $s$ from $\bar{w}<1.1$, and for the template background $b$ from $1.3<\bar{w}<1.5$. A necessary correction applied to the template background accounts for differences in radial profile between the two $\bar{w}$ regimes. A normalisation $\alpha$, to derive excess events $s-\alpha b$ at some position in the FOV, accounts for differences in the total number of events in the two $\bar{w}$ regimes. A full description of the template model appears in Rowell (2002). Figure 1 presents the resulting excess skymap. The template model was used in discovering the $\mathrm{TeV}$ source which is evident $\sim 0.5^{\circ}$ north of Cyg X-3. An event-by-event centre of gravity (COG) calculation (Table 1a), weighting events with \pm 1 from the $s$ and $\alpha b$ regimes respectively is performed. The COG accuracy is limited by a systematic pointing error of $25^{\prime \prime}$ (Pühlhofer et al. 1997). A pre-trial significance at the COG position of $+5.9 \sigma$ is obtained, summing events within $\theta=0.12^{\circ}$ (Table $1 \mathrm{~b}$ ). Statistical trial factors arise from the initial "discovery" skymap (different to that in Fig. 1) in which event directions are independently summed in 1100 bins of size $0.1^{\circ} \times 0.1^{\circ}$. Assuming 1100 trials are accrued in locating the COG, the post-trial probability $P_{\mathrm{t}}=1.0-(1-P)^{1100}$ for $P$ the pre-trial probability (one-sided $P=1.9 \times 10^{-9}$, or $+5.9 \sigma$ ), is then calculated as $P_{\mathrm{t}}=2.1 \times 10^{-6}$. This gives a post-trial significance of $+4.6 \sigma .1100$ is actually a slightly conservative trial estimate since oversampling of the $\gamma$-ray point spread function (PSF) by a factor $\sim 1.5$ occurs in the discovery skymap. To verify results using the template model, we make use of a conventional type of background model employing background regions displaced from the on-source region spatially in the FOV

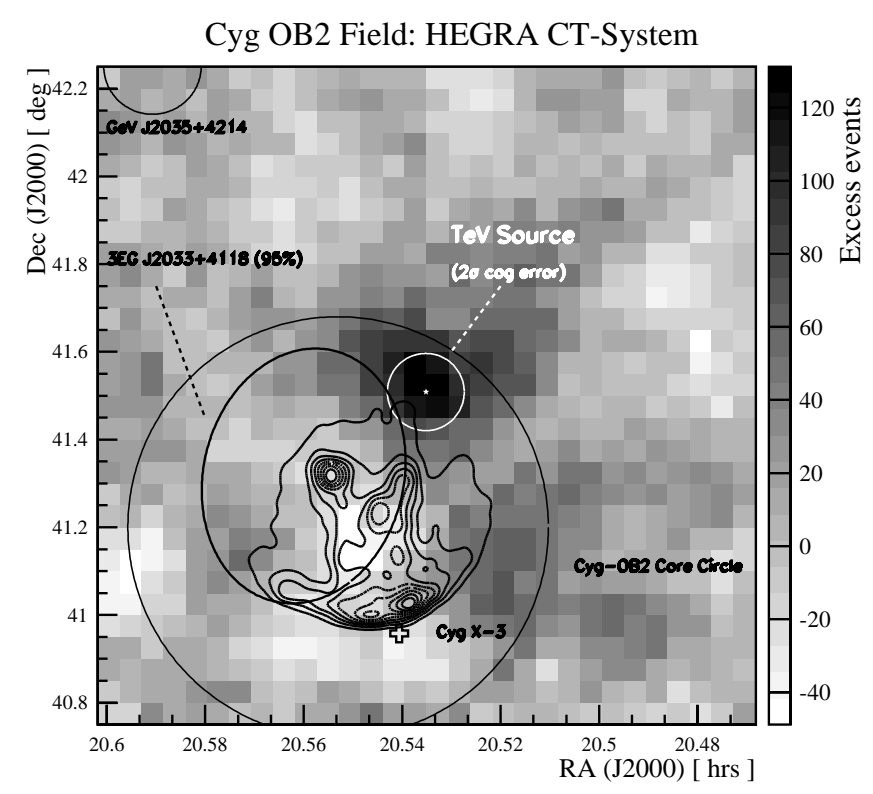

Fig. 1. Skymap $\left(1.5^{\circ} \times 1.5^{\circ}\right.$ view at $0.05^{\circ} \times 0.05^{\circ}$ binning $)$ of excess events $s-\alpha b$, using the template background model. At each bin, the excess is estimated from events within a radius $\theta=0.12^{\circ}$. Included are $95 \%$ error ellipses of various EGRET sources, the core of Cygnus OB2 (Knödlseder 2000), the TeV COG (star), its $2 \sigma$ error circle, and the location of Cyg X-3. ASCA GIS contours (2-10 keV) are overlayed.

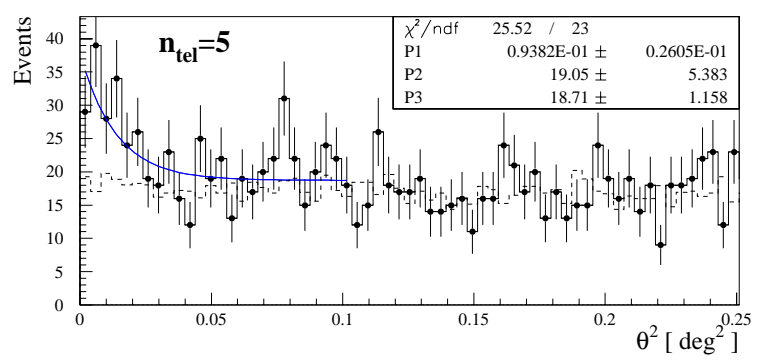

Fig. 2. Distribution of $\theta^{2}$ for $\theta$ calculated from the COG (solid histogram and filled dots), against a background derived from the template model (dashed hist). The convolved radial Gaussian fit $F=$ $P 3+P 2 \exp \left(-\theta^{2} /\left(P 1^{2}+\sigma_{\mathrm{pt}}^{2}\right)\right)$ is indicated by the solid line with $P 1=\sigma_{\text {src }}=0.094^{\circ} \pm 0.026^{\circ}$ the intrinsic source size. The PSF width $\sigma_{\mathrm{pt}}=0.070^{\circ}$ is estimated from Crab data.

but derived from the same $\bar{w}<1.1$ regime. Background events are taken from ring-segments with matching trigger characteristics to that of the source region. A normalisation $\alpha$ according to the solid angle ratio between background and on-source regions is then applied. Results using this so-called ring model (Table $1 b$ ) are consistent with those from the template model.

\subsection{Observational properties of the TeV source}

Splitting data firstly according to their three tracking positions reveals commensurate source contributions (Table 1c). The source is also found to develop linearly with the cumulative number of background events. Such tests suggest consistency with a steady source during the three years of data collection. We have also verified that after cuts a constant 
Table 1. Summary of numerical results for the TeV source, under two background models. Here, $s$ and $b$ are the resulting event numbers for the $\gamma$-ray-like and background $\bar{w}$ regimes respectively, and $s-\alpha b$ is the derived excess using a normalisation $\alpha . S$ denotes the excess significance using Eq. (17) of Li \& Ma (1983). See Sect. 2 for definitions of $\theta$ and $\bar{w}$.

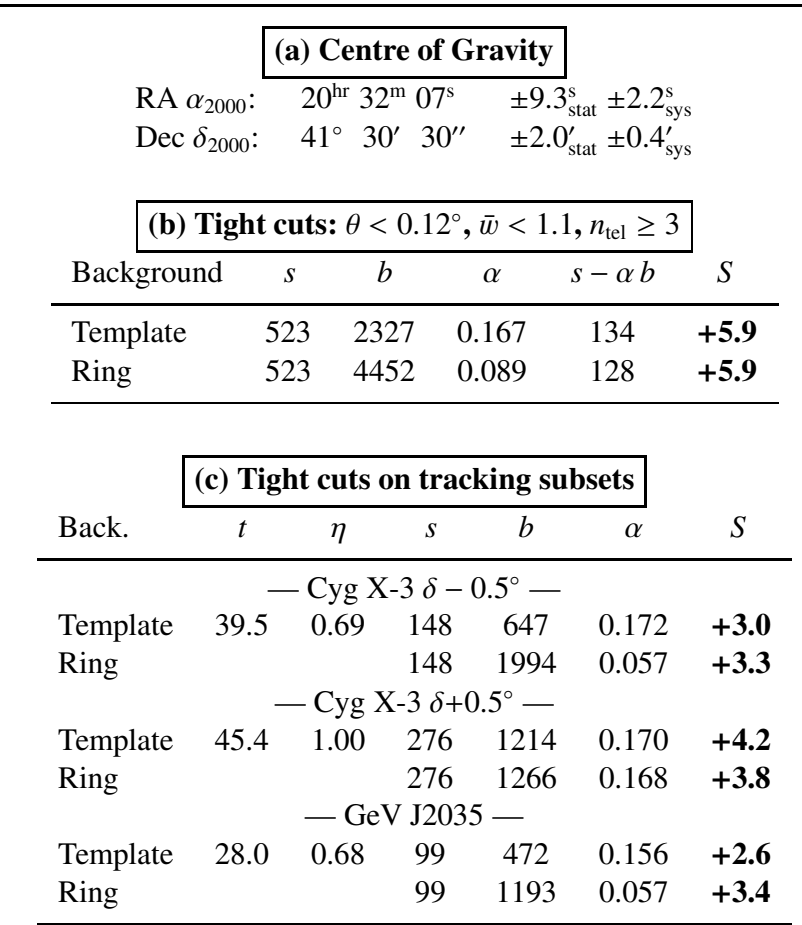

$t$ : Observation time (hrs)

$\eta$ : Estimated $\gamma$-ray trigger effic. $c f$. on-axis.

\begin{tabular}{|c|c|c|c|c|c|}
\hline \multirow[b]{2}{*}{ Back. } & \multicolumn{4}{|c|}{ (d) Tight cuts on $n_{\text {tel }}$ subsets } & \multirow[b]{2}{*}{$S$} \\
\hline & $s$ & $b$ & $\alpha$ & $\overline{s-\alpha} b$ & \\
\hline \multicolumn{6}{|c|}{$-n_{\mathrm{tel}}=2-$} \\
\hline Template & 387 & 865 & 0.433 & 12 & +0.8 \\
\hline Ring & 387 & 4619 & 0.082 & 8 & +0.5 \\
\hline \multicolumn{6}{|c|}{$-n_{\mathrm{tel}}=3-$} \\
\hline Template & 272 & 904 & 0.224 & 70 & +4.1 \\
\hline Ring & 272 & 2691 & 0.086 & 41 & +2.6 \\
\hline \multicolumn{6}{|c|}{$-n_{\text {tel }}=4-$} \\
\hline Template & 133 & 774 & 0.130 & 32 & +2.9 \\
\hline Ring & 133 & 1110 & 0.088 & 44 & +3.2 \\
\hline \multicolumn{6}{|c|}{$-n_{\mathrm{tel}}=5-$} \\
\hline Template & 118 & 777 & 0.089 & 50 & +5.1 \\
\hline Ring & 118 & 651 & 0.102 & 52 & +5.4 \\
\hline
\end{tabular}

\begin{tabular}{|c|c|c|c|c|c|}
\hline \multicolumn{6}{|c|}{ (e) Spectral Cuts ${ }^{\dagger}: \theta<0.224^{\circ}, \bar{w}<1.1, n_{\text {tel }} \geq 3$} \\
\hline Back. & $s$ & $b$ & $\alpha$ & $s-\alpha b$ & $S$ \\
\hline Ring & 366 & 3222 & 0.087 & 86 & +4.7 \\
\hline
\end{tabular}

background acceptance throughout the dataset is observed and that the event excess in $\bar{w}$-space appears consistent with that of a true $\gamma$-ray population. To determine source size, we fit a radial Gaussian convolved with the point spread function

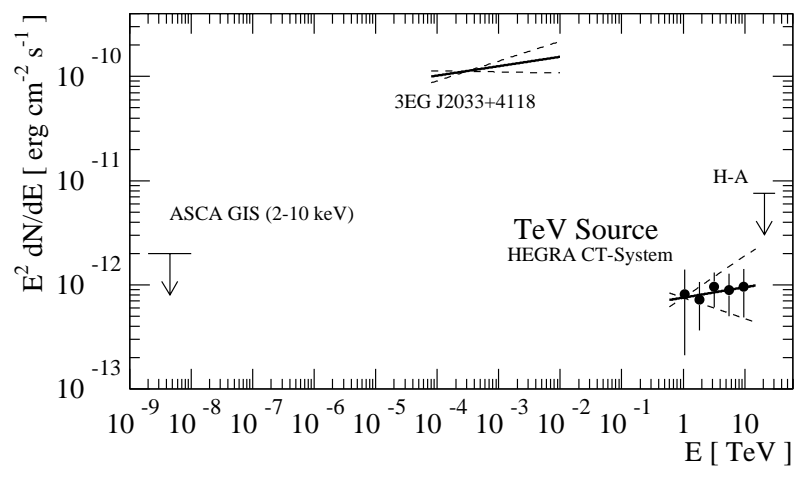

Fig. 3. Differential energy fluxes of the $\mathrm{TeV}$ source and other results. "H-A" is the AIROBICC 90\% confidence level upper limit (Prahl 1999) at the TeV COG converted to differential form at $20.8 \mathrm{TeV}$ assuming a spectral photon index of -2.0 . We interpret the 3EG J2033+4118 flux as an upper limit. The ASCA GIS 99\% upper limit assumes a photon index of 2, and $N_{\mathrm{H}}=10^{22} \mathrm{~cm}^{-2}$. Data fits (solid lines) and their $1 \sigma$ statistical errors in photon index (dashed lines) are shown. $\mathrm{TeV}$ data are well fit $\left(\chi_{v}^{2}=0.23 / 3\right)$ by a power law (Eq. (1)).

(determined from Crab data) to the excess events as a function of $\theta^{2}$, using a subset of events with the best angular resolution $\left(n_{\text {tel }}=5\right)$ for which errors are minimised (Fig. 2). The intrinsic size of the $\mathrm{TeV}$ source is estimated at $\sigma_{\mathrm{src}}=5.6^{\prime} \pm 1.7^{\prime}$. Correlations between the fit parameters suggest that the significance for a non-zero source size is at the $\sim 3.0 \sigma$ level rather than the $3.5 \sigma$ level indicated above. A breakdown of the excess with $n_{\text {tel }}$ also shows that the $n_{\text {tel }}=5$ exclusive subset contributes strongly to the excess (Table 1d). Such behaviour is suggestive of a generally hard spectral index given that higher trigger multiplicities are favoured by higher energy events. For the energy spectrum calculation and selection cuts, we follow the method of Aharonian et al. (1999) using effective collection areas appropriate for on-axis and $\sim 1^{\circ}$ off-axis sources as per the exposure efficiency for the $\mathrm{TeV}$ source in these data. A tight cut $\bar{w}<1.1$, as opposed to the less-restrictive $\bar{w}<1.2$ is also used. For energies below $\sim 0.8 \mathrm{TeV}$ the effective collecting area decreases markedly sharper at positions beyond $\sim 1.0^{\circ}$ off-axis compared to positions nearer on-axis. Limiting our fit therefore to energies $>0.8 \mathrm{TeV}$ reduces systematic errors. A socalled loose cut $\theta<0.224^{\circ}$ using the ring background model is used in deriving the energy bin-by-bin excess since a loose cut in $\theta$ improves the $\gamma$-ray selection efficiency according to the moderately-extended nature of the source. Results are shown in Table 1e and Fig. 3, with the spectrum being well fit by a pure power law with generally hard photon index. Systematic errors are estimated from changes in bin centres and uncertainties in Monte Carlo-derived collection areas:

$$
\begin{aligned}
\mathrm{d} N / \mathrm{d} E & =N(E / 1 \mathrm{TeV})^{\gamma} \mathrm{ph} \mathrm{cm}^{-2} \mathrm{~s}^{-1} \mathrm{TeV}^{-1} \\
N & =4.7\left( \pm 2.1_{\text {stat }} \pm 1.3_{\text {sys }}\right) \times 10^{-13} \\
\gamma & =-1.9\left( \pm 0.3_{\text {stat }} \pm 0.3_{\text {sys }}\right) .
\end{aligned}
$$

The integral flux at $F(E>1 \mathrm{TeV})=4.5\left( \pm 1.3_{\text {stat }}\right) \times$ $10^{-13} \mathrm{ph} \mathrm{cm}^{-2} \mathrm{~s}^{-1}$ amounts to $2.6 \%$ that of the Crab. 


\section{Discussion and conclusion}

The OB association Cygnus OB2 is unique for its compact nature and extreme number of member $\mathrm{OB}$ and $\mathrm{O}$ stars (e.g. Knödlseder 2000), and both theoretical and observational grounds for non-thermal particle acceleration have longbeen discussed (e.g. Montmerle 1979; Cassé \& Paul 1980; Völk \& Forman 1982; White \& Chen 1992). The TeV source is positioned inside the core of Cygnus OB2 as defined by Knödlseder (2000). Assuming the $\mathrm{TeV}$ source is as distant as Cygnus OB2 $(1.7 \mathrm{kpc})$, a luminosity $\sim 10^{32} \mathrm{erg} \mathrm{s}^{-1}$ above $1 \mathrm{TeV}$ is implied, well within the kinetic energy (KE) budget of Cygnus OB2 estimated recently by Lozinskaya et al. (2002) at a few $\times 10^{39} \mathrm{erg} \mathrm{s}^{-1}$, and also within the KE budget of a number of notable member stars (e.g. Massey \& Thompson 1991; Manchanda et al. 1996; Benaglia et al. 2001). So far no counterparts at other wavelengths are identified. No massive or luminous Cygnus OB2 star of note discussed recently (e.g. Massey \& Thompson 1991; Romero et al. 1999; Herrero et al. 2001; Benaglia et al. 2001) is positioned within the $1 \sigma \mathrm{TeV}$ error circle. No catalogued X-ray source from the ROSAT all-sky and pointed survey lies within the $2 \sigma \mathrm{TeV}$ error circle. Our analysis of archival ASCA GIS data yields a 99\% upper limit (2$10 \mathrm{keV}$ ) of $2.0 \times 10^{-12} \mathrm{erg} \mathrm{cm}^{-2} \mathrm{~s}^{-1}$ (Fig. 3). Such results may imply that the energy source for particle acceleration is not colocated with the TeV source, arising instead from the winds of the young/massive stars of Cygnus OB2, either individually or collectively, or from an alternative source. The former scenario would generally favour accelerated hadrons interacting with a local, dense gas cloud, giving rise to $\pi^{\circ}$-decay $\mathrm{TeV}$ emission. The likely hard $\mathrm{TeV}$ spectrum can be explained by (energydependent) diffusion effects, accelerator age, and accelerator to target distance (see e.g. Aharonian 2001). There is however at present no strong indication from $\mathrm{CO}$ and HII surveys (Leung \& Thaddeus 1992; Dame et al. 2001; Comeron \& Torra 2001) for any co-located dense gas cloud, although see Martí et al. (2000) who discuss a nearby HII cloud in the context that follows immediately below. A suggested alternative scenario involves a jet-driven termination shock at which accelerated electrons produce synchrotron and $\mathrm{TeV}$ inverse-Compton (IC) emission (Aharonian \& Atoyan 1998). Such a jet could emanate from a nearby microquasar, possibly a class of high energy $\gamma$-ray source (see e.g. Paredes et al. 2000). In fact two nearby sources, 3EG J2033+4118 and also the EGRET source possibly associated with Cyg X-3 (Mori et al. 1997) could be $\mathrm{GeV}$ indicators of such a microquasar. Remarkably, Cyg X-3 appears to have a bi-lobal jet (Martí et al. 2000, 2001) wellaligned with the TeV source, the latter which would be $\sim 70 \mathrm{pc}$ from Cyg X-3 in absolute terms if it is at the same distance $(>8.5 \mathrm{kpc})$. Future X-ray observations will be a crucial constraint on the IC emission in this context. We interpret the flux from 3EG J2033+4118 presently as a MeV/GeV upper limit at the TeV COG (Fig. 3), and note that the directly extrapolated energy flux from the TeV source lies about two orders of magnitude below the 3EG J2033+4118 flux at overlapping energies. Finally we note that earlier claims for a $\mathrm{TeV}$ source (Neshpor et al. 1995, at a $\sim 1$ Crab flux level) and flaring episodes coincident with a Cyg X-3 radio flare at energies >40 TeV (Merck 1993; Krawczynski 1995) positionally consistent with our TeV COG have been reported. These results are however in conflict with our estimates of the flux level and steady nature of the $\mathrm{TeV}$ source assuming they all have the same origin. Further observations with the HEGRA CT-System aimed at confirmation and improving our spectral and source morphology studies are now underway.

Acknowledgements. The support of the German ministry for Research and technology BMBF and of the Spanish Research Council CICYT is gratefully acknowledged. We thank the Instituto de Astrofísica de Canarias for the use of the site and for supplying excellent working conditions at La Palma. We gratefully acknowledge the technical support staff of the Heidelberg, Kiel, Munich, and Yerevan Institutes. GPR acknowledges receipt of a von Humboldt fellowship.

\section{References}

Aharonian, F. A., \& Atoyan, A. M. 1998, New. Astron. Rev., 42, 579 Aharonian, F. A., Akhperjanian, A. G, Barrio, J. A., et al. 1999, A\&A, 349,11

Aharonian, F. A. 2001, Space. Sci. Rev., 99, 187

Benaglia, P., Romero, G. E., Stevens, I. R., \& Torres, D. F. 2001, A\&A, 366, 605

Cassé, M., \& Paul, J. A. 1980, ApJ, 237, 236

Comeron, F., \& Torra, J. 2001, A\&A, 375, 539

Dame, T. M., Hartmann, D., \& Thaddeus, P. 2001, ApJ, 547, 792

Daum, A., Herman, G., Hess, M., et al. 1997, Astropart. Phys., 8, 1

Herrero, A., Puls, J., Corral, L. J., et al. 2001, A\&A, 366, 623

Hofmann, W., Jung, I., Konopelko, A., et al. 1999, Astropart. Phys., 10,275

Knödlseder, J. 2000, A\&A, 360, 539

Konopelko, A. 1995, in Proc. Towards a Major Čerenkov Detector IV (Padova), 373

Konopelko, A., Hemberger, M., Aharonian, F. A., et al. 1999 , Astropart. Phys., 10, 275

Krawczynski, H. 1995, Diploma Thesis, University of Hamburg

Lamb, D. Q., \& Macomb, D. J. 1997, ApJ, 488, 872

Leung, H. O., \& Thaddeus, P. 1992, ApJS, 81, 267

Li, T. P., \& Ma, Y. Q. 1983, ApJ, 272, 317

Lozinskaya, T. A., Pravdikova, V. V., \& Finoguenov, A. V. 2002, Astron. Lett., 28, 223

Manchanda, R. K., Polcaro, V. F., Norci, L., et al. 1996, A\&A, 305, 457

Martí, J., Paredes, J. M., \& Peracaula, M. 2000, ApJ, 545, 939

Martí, J., Paredes, J. M., \& Peracaula, M. 2001, A\&A, 375, 476

Massey, P., \& Thompson, A. B. 1991, AJ, 101, 1408

Merck, M. 1993, Ph.D. Dissertation, Ludwig-Maximilians University Munich

Montmerle, T. 1979, ApJ, 231, 95

Mori, M., Bertsch, D. L., Dingus, B. L., et al. 1997, ApJ, 476, 842

Neshpor, Y. I., Kalekin, O. R., Stepanian, A. A., et al. 1995, Proc. 24th ICRC (Rome) 2, 1385

Paredes, J. M., Martí, J., Ribo, M., \& Massi, M. 2000, Science, 288, 2340

Prahl, J. 1999, Ph.D. Dissertation, Universität Hamburg

Pühlhofer, G., Daum, A., Hermann, G., et al. 1997, Astropart. Phys., 8,11

Romero, G. E., Benaglia, P., \& Torres, D. F. 1999, A\&A, 348, 868

Rowell, G. P. 2002, in preparation

Völk, H. J., \& Forman, M. 1982, ApJ, 253, 188

White, R. L., \& Chen, W. 1992, ApJ, 387, L81 